\title{
Anabases
}

ANABASES Traditions et réceptions de l'Antiquité

$11 \mid 2010$

Varia

\section{Erreurs à Delphes. La tholos de Marmaria au fil des interprétations (ca 1840-1940)}

Nicolas Kyriakidis

\section{(2) OpenEdition}

Journals

Édition électronique

URL : http://journals.openedition.org/anabases/836

DOI : 10.4000/anabases.836

ISSN : 2256-9421

Éditeur

E.R.A.S.M.E.

Édition imprimée

Date de publication : 1 mars 2010

Pagination : 149-163

ISSN : 1774-4296

\section{Référence électronique}

Nicolas Kyriakidis, « Erreurs à Delphes. La tholos de Marmaria au fil des interprétations (ca

1840-1940) », Anabases [En ligne], 11 | 2010, mis en ligne le 01 mars 2013, consulté le 20 octobre

2019. URL : http://journals.openedition.org/anabases/836 ; DOI : 10.4000/anabases.836

(c) Anabases 
Anabases 11 (2010), p. 149-163.

\section{Erreurs à Delphes. La tholos de Marmaria au fil des interprétations $(c a \text { 1840-1940 })^{1}$}

NicOlas KYRIAKIDIS

Depuis sa première déCouverte en 1836, la tholos du sanctuaire d'Athéna Pronaia à Delphes, au lieu-dit Marmaria, édifice aussi exceptionnel que mystérieux, a attiré l'attention des archéologues et a suscité une abondante littérature savante. Des hypothèses, dont nombre sont aujourd'hui tenues pour erronées, ont été proposées pour sa restitution, son affectation et sa datation, jusqu'à ce qu'on parvienne au début des années 1940 à un état de la recherche que l'on peut qualifier de contemporain - quoique la connaissance du bâtiment ait significativement progressé ces dernières années ${ }^{2}$.

1 J'adresse tous mes remerciements à $S$. Rey qui m'a offert l'opportunité de me pencher sur un monument des plus riches pour qui veut étudier l'historiographie de la recherche à Delphes. Ma reconnaissance va à Mmes A. Jacquemin, C. Bonnet et H. Brun-Siard ainsi qu'à M.D. Laroche qui m’ont guidé de leurs conseils. Toutes les imperfections de ce texte sont de mon fait.

2 Pour un état actuel des connaissances et une première approche, cf. J.-F. BOMMELAER, D. Laroche, Guide de Delphes. Le site, 1991, nº 40 et J.-F. Bommelaer (éd.), Marmaria. Le sanctuaire d'Athéna à Delphes, 1997, passim. Principales contributions depuis 1940: G. Roux, "Le toit de la tholos de Marmaria et la couverture des monuments circulaires grecs ", Bulletin de correspondance hellénique 76 (1952), p. 442-483; J. BOUSQUET, "La destination de la Tholos de Delphes", Revue historique 223 (1960), p. 287-298; G. Roux, “Pausanias, le 'Contre Aristogiton' et les 'Énigmes de Marmaria' à Delphes”, Revue des Études anciennes 67 (1965), p. 37-48; S. SeTtIS, "Un enigma delfico, Pausania, la tholos e il Phylakeion”, Annuario della Scuola Archeologica di Atene 45-46 (1967-1968), p. 355-377; G. Roux, Delphes, son oracle et ses dieux, 1976, p. 191-192; J. MarcadÉ, "Les métopes mutilées de la tholos de Marmaria à Delphes", Comptes rendus des séances de l'Académie des inscriptions et belles lettres (1979), p. 151-170, p. 151-170; J. BOusqueT, "Réflexions 
Cet édifice, remarquable à plusieurs égards, se prête donc particulièrement bien à une enquête historiographique dans le cadre d'une journée d'étude consacrée à l'erreur dans les Sciences de l'Antiquité.

L'intérêt que l'on porte à la tholos s'explique pour plusieurs raisons. Le bâtiment se singularise tout d'abord par l'originalité de son plan, la rotonde, rare dans l'architecture grecque. Le défi que représente la conception de l'édifice a justifié que son architecte lui ait consacré un livre dont le titre seul nous est conservé par Vitruve ${ }^{3}$. Le coût de sa construction a été très important car les matériaux mis en œuvre (marbre pentélique et calcaire sombre d'Éleusis) ont été importés d'aussi loin qu'Athènes; la qualité de la finition ensuite est des plus remarquables; ce bâtiment s'est vu adjoindre un décor sculpté. Toutes ces caractéristiques contribuent à en faire un monument « de prestige " dès l'Antiquité. Sa réputation n'a fait que s'amplifier chez les Modernes. Ainsi, lorsque Th. Homolle informe l'Académie des inscriptions et belles lettres de sa redécouverte en 1901, il précise que " la tholos [...] est plus ancienne, plus riche avec simplicité et plus parfaite d'exécution que les édifices analogues d'Épidaure ou d'Olympie $^{4}$ » et la qualifie peu après de "fin bijou de l'architecture antique ${ }^{5}$ ». Pour É. Bourguet, elle est « un des chef d'œuvres les plus illustres [...] un de ces rares modèles que l'on admire dès le premier regard, mais pour lesquels l'admiration grandit à mesure qu'on les connaît mieux ${ }^{6}$ ». Pourtant, ce monument est très mal documenté: aucune inscription ne nous informe sur les commanditaires de l'ouvrage ou sur sa

sur la Tholos de Delphes", Revue des Études grecques 93 (1980), p. X-XI ; L. LerAT, "Les "énigmes de Marmaria”, Bulletin de correspondance hellénique 109 (1985), p. 255-264; F. SEILER, Die griechische Tholos. Untersuchungen zur Entwicklung, Typologie und Funktion kunstmässiger Rundbauten, 1986, p. 56-71; G. Roux, "La tholos d'Athéna Pronaia dans son sanctuaire de Delphes", Comptes rendus des séances de l'Académie des inscriptions et belles lettres (1988), p. 290-309 et 835-839; G. Roux, "Une querelle de préséance à Delphes: la promantie des Tarentins et des Thouriens", Zeitschrift für Papyrologie und Epigraphik 80 (1990), p. 23-29; D. Laroche, "La Tholos de Delphes: formes et destination", in J.-F. Bommelaer (éd.), Delphes, centenaire de la " grande fouille " de l'École française d'Athènes (1892-1903), Actes du colloque Paul Perdrizet, Strasbourg 6-9 novembre 1991, 1992, p. 207-223; J. Bousquet, "La tholos de Delphes et les mathématiques préeuclidiennes", Bulletin de correspondance hellénique 117 (1993), p. 285-313; J.-F. BOMMELAER, "Sur quelques nouveautés de l'architecture du IV siècle", in P. CARLIER (éd.), Le IV siècle av. J.-C. Approches historiographiques, Actes du colloque Nancy 1994, 1996, p. 141-151; W. Hoepfner, "Zur Tholos in Delphi”, Archäologische Anzeiger (2000), p. 99-107 [cf. Bull. analyt. d'arch. 2002, 228]. Pour ce qui a trait à l'histoire de la recherche à Delphes, cf. Collectif, La redécouverte de Delphes, 1992.

3 Vitruve, VII, préf. 12.

4 Th. Homolle, "Séance du 23 octobre 1901", Comptes rendus des séances de l'Académie des inscriptions et belles lettres (1901), p. 639.

5 Th. Homolle, "Fouilles de Delphes. Les découvertes de Marmaria", Revue de l'art ancien et moderne 15 (1904), p. 20.

6 É. Bourguet, Les ruines de Delphes, 1914, p. 320. 
destination, aucun texte non plus à part le passage allusif de Vitruve. Il semble bien que, pour une raison inconnue qui n'a cessé d'étonner les archéologues, Pausanias n'ait pas mentionné la tholos dans sa description du sanctuaire d'Athéna Pronaia ${ }^{7}$. Ne pouvant accepter ce silence pour un monument qui leur semblait parmi les plus dignes d'être notés et commentés, les archéologues ont voulu le retrouver dans des bâtiments cités par Pausanias ou mentionnés dans des inscriptions, sans qu'aucune proposition ne parvienne à faire l'unanimité.

Ce silence des auteurs anciens ou notre incapacité à repérer les éventuelles mentions textuelles de la tholos expliquent, pour une part, l'abondance de la bibliographie la concernant: on compte une cinquantaine de références, dont plusieurs monographies, qui lui sont entièrement consacrées. C'est à cette production savante, professionnelle, à portée scientifique que je bornerai mon étude en m'intéressant aux scories de la recherche: les hypothèses ou les interprétations erronées. La production scientifique sur ce bâtiment s'articule autour de trois questions, traditionnelles en architecture mais ici particulièrement ardues: celle de la restitution architecturale de l'édifice, celle de sa datation, celle enfin de sa fonction. Ces trois questions entretiennent des relations complexes: si la datation d'une part et la fonction de l'autre sont relativement indépendantes, elles sont chacune liées à la restitution. C'est pour répondre à ces interrogations que les archéologues ont sollicité les sources textuelles et matérielles en rapport avec ce monument.

Sans chercher d'emblée à définir le concept d'erreur, qui pose problème dans le domaine des Sciences de l'homme où, bien souvent, les résultats se placent dans le champ de la vraisemblance ou de la conviction, je me contenterai d'une acception pour ainsi dire " historiographique ": est erroné ce qui n'a plus cours, ce qui n'est plus jugé actuel par la communauté des professionnels de la discipline. S'agissant de la tholos de Delphes, on peut analyser le fonctionnement particulier de ces errances, tant pour ce qui relève de la mise en œuvre du raisonnement scientifique que pour le contexte historique déterminé - et souvent déterminant - dans lequel il se développe.

\section{Les textes et l'identification des vestiges}

Lorsque l'architecte Laurent, Allemand de Dresde au service du jeune État grec, fut envoyé à Kastri (nom moderne de Delphes) en octobre 1838 pour des affaires de cadastre, il se saisit de l'occasion pour mener des fouilles d'ampleur limitée au lieu-dit Marmaria. Le fruit de ses travaux nous est parvenu grâce à son compatriote l'universitaire H.N. Ulrichs qui prit soin de les faire connaittre en même temps qu'il publiait en 1840 le récit de son propre voyage archéologique ${ }^{8}$. Ulrichs présente quatre monuments

7 Pausanias, X, 8, 6-8.

8 H.N. ULRICHS, Reisen und Forschungen, 1840, p. 263-264. 
fouillés ou plutôt sondés par Laurent sur la terrasse du sanctuaire d'Athéna à Marmaria. Seule la tholos retint son attention, tant pour son état de conservation que pour la qualité du travail des blocs de l'élévation. Dès lors, l'attribution semblait acquise: le plus beau monument est forcément le plus important dans la tradition textuelle. Il ne pouvait donc s'agir que du temple d'Athéna Pronaia, connu par des mentions d'Hérodote $^{9}$, de Démosthène ${ }^{10}$ et de Pausanias ${ }^{11}$. Et si les vestiges n'étaient pas assez loquaces, il semblait parfaitement licite de les faire parler grâce aux textes. Hérodote et Pausanias mentionnent le pronaos du temple d'Athéna. Ce qui est banal pour un édifice rectangulaire est très rare pour une rotonde. Comment imaginer une rotonde à pronaos? Un exemple prestigieux offre un modèle tout trouvé: le panthéon dit d'Agrippa à Rome. C'est donc à sa ressemblance qu'Ulrichs proposa de restituer la tholos, hors de toute preuve matérielle. Les tambours trouvés en fouille furent interprétés comme étant ceux du pronaos, et de tenter de faire correspondre les fragments de statues découverts avec ceux des œuvres citées par les auteurs antiques.

Plus d'une génération après, dans les années 1860, P. Foucart, premier membre de l'École française d'Athènes à entreprendre des travaux d'importance à Delphes, visita Marmaria. Force lui fut de constater qu' " il est impossible d'y rien reconnaitre ${ }^{12}$ ". Les vestiges ont tellement été perturbés que l'interprétation n'en est plus possible. P. Foucart fut contraint de paraphraser, en la citant, la présentation d'Ulrichs. Il alla cependant plus loin que lui en datant, explicitement, la tholos d'avant Hérodote, c'està-dire, au plus tard, de la fin de l'époque archaïque. Une de ses remarques est particulièrement intéressante. Il nota la différence d'appareil entre le mur de terrasse, polygonal à joints courbes qui est daté aujourd'hui du $\mathrm{VI}^{\mathrm{e}} \mathrm{s}$. et qu'il attribue à une " haute antiquité ", et celui de la tholos. Plutôt que de remettre en cause l'identification entre le beau temple des textes et les beaux vestiges de la fouille, il supposa alors l'existence d'un état antérieur, contemporain de la terrasse, les vestiges encore visibles, c'est-à-dire la tholos, restant interprétés comme le temple de la fin de l'archaïsme vu par Hérodote. Or, l'étude architecturale postérieure a établi que, pour des raisons de datation, cette identification était impossible ${ }^{13}$. Cet exemple montre le danger, permanent, qu'il y a à voir le terrain et les vestiges à travers le prisme déformant des sources textuelles au mépris de l'observation.

Achevons sur une dernière considération pour montrer comment l'on passe de l'erreurà la faute. Ulrichs donne dans son ouvrage un plan levé par Laurent. Les vestiges de Marmaria y sont peu visibles, car il s'agit d'un relevé (cf. infra fig. 1). Ulrichs récupère le fond de carte pour y restituer les monuments antiques (cf. infra fig. 2). Cette

9 Hérodote, I, 92.

10 Démosthène, Contre Aristogiton, I, 34.

11 Pausanias, X, 8, 6-8.

12 P. Foucart, Mémoire sur les ruines et l'histoire de Delphes, 1865, p. 12-13.

13 C'est le "temple en tuf " qui est interprété comme le temple d'Athéna de la fin de l'archaïsme. Cf. J.-F. Bommelaer, D. Laroche, Guide de Delphes, nº 29. 
rotonde si belle promue au rang de temple d'Athéna se doit d' " accrocher " le regard du lecteur. Si l'on se fie à l'échelle de son plan, c'est un monument de près de $25 \mathrm{~m}$ de diamètre. Or, le diamètre de la tholos est de $13,50 \mathrm{~m}^{14}$ au dernier degré de la krépis. Approximation normale pour un plan à si petite échelle? Dessin au hasard en l'absence de relevé précis, comme semble l'indiquer l'absence de toute donnée chiffrée dans son rapport des activités de Laurent? Volonté de faire correspondre l'ampleur topographique à l'importance littéraire? En tout cas, à identification fausse, plan faux.

\section{Restitution et datation: la colonnade extérieure et la datation du monument}

Les fouilleurs français qui mettent au jour les monuments et leur attribuent les fragments ainsi découverts se servent de deux critères - les plus évidents car les mieux étudiés à l'époque - pour dater la tholos. Le premier est le profil de l'échine de son chapiteau dorique, le second, le style des métopes sculptées. Th. Homolle place les premiers dans la continuité du Parthénon (achevé pour l'essentiel en 438), les seconds entre l'Érechtheion et le Mausolée (fin $v^{\mathrm{e}}$ et $1^{\mathrm{re}}$ moitié du IV s. $)^{15}$. Avec les sculptures d'Épidaure, invoquées par É. Bourguet ${ }^{16}$, qui, lui, situe la tholos au premier tiers du $\mathrm{IV}^{\mathrm{e}}$ s., les principaux parallèles sont trouvés. Dès lors, il s'agit de trancher: " fin $\mathrm{V}^{\mathrm{e}}$ ou début $\mathrm{IV}^{\mathrm{e}}{ }^{17}$ ? La question est plus importante qu'il peut sembler à première vue: cela fait d'abord une bonne génération d'écart; mais, surtout, il s'agit de savoir si, dans l'histoire de l'art et de l'architecture, la tholos tient plus du style parthénonien, de la haute période qui est la bonne période, ou s'il faut la ranger au $\mathrm{IV}^{\mathrm{e}} \mathrm{s}$. parmi les prodromes $\mathrm{du}$ second classicisme.

Les deux premières études globales du monument sont celle de $\mathrm{H}$. Pomtow et de son architecte H. Wenzel, publication " pirate " parue dans la revue Klio en $1912^{18}$ et celle de J. Charbonneaux et K. Gottlob, la publication officielle, dans la série des Fouilles de Delphes en $1925^{19}$. Ces deux études, concurrentes et de qualité bien inégale, ont eu à relever le défi de la restitution de l'élévation d'une tholos qui n'était connue qu'en plan. La seule certitude que l'on pouvait tirer des tambours de colonne, qui avaient beaucoup souffert et se laissaient mal mesurer, était qu'ils étaient tous de

\footnotetext{
14 J.-F. Bommelaer (éd.), Marmaria..., p. 59.

15 Th. Homolle, "Séance du 23 octobre 1901", p. 639.

16 É. Bourguet, Les ruines de Delphes, p. 320.

17 Rappelons que la tholos est aujourd'hui datée vers 380-370, cf. J.-F. BommelaER, D. Laroche, Guide de Delphes, n' 40.

18 H. R. Pomtow, "Die grosse Tholos zu Delphi und die Bestimmung der delphischen Rundbauten”, Klio 12 (1912), p. 179-218 et 281-307.

19 J. Charbonneaux, FD II 4, Le sanctuaire d'Athéna Pronaia. La Tholos, 1925.
} 
la même hauteur, ce qui est exceptionnel ${ }^{20}$. Dès lors que le chapiteau de la colonne orientait vers la fin du $v^{e} s$., la question du nombre de tambours ne semble même pas s'être posée: tant Pomtow et Wenzel ${ }^{21}$ que Charbonneaux et Gottlob ${ }^{22}$ restituèrent sans hésiter quatre tambours à la colonne, soit une hauteur de 4,813 m (chapiteau compris), avec un diamètre inférieur à $0,868 \mathrm{~m}$. On obtenait ainsi la proportion de référence de 5,54 (hauteur de la colonne/diamètre inf.). Cette proportion prenait place dans une série bien étudiée, les colonnes devenant plus élancées avec le temps : 5,5 au Parthénon; 6,09 au temple d'Athéna Aléa à Tégée (ca 345-335); 6,64 au temple de Zeus à Némée (ca 330-320 23. Comme l'écrit H. Pomtow, c'est la hauteur normale, attendue pour la période ${ }^{24}$ et en retour, la restitution justifie la datation de ce bâtiment d'aspect post-parthénonien ${ }^{25}$. Dans une fourchette de datation qui va de la fin du v $v^{e}$ s. au milieu du IV $V^{\mathrm{e}}$, la proportion des colonnes s'ajoute au profil des chapiteaux et à la modénature des moulures qui tous vont dans le sens d'une datation haute. C'est à partir de ces éléments "nobles " du bâtiment que J. Charbonneaux défend la datation la plus haute possible: dernier quart $\mathrm{du} \mathrm{v}^{\mathrm{e}} \mathrm{s}^{26}$. Par attraction, il considère que les sculptures permettent une datation au dernier quart $\mathrm{du} \mathrm{v} / \mathrm{v}^{\mathrm{er}}$ quart $\mathrm{du} \mathrm{IV}^{\mathrm{e}}$, alors qu'elles passaient plutôt comme étant du $1^{\text {er }}$ tiers du IV .

Cette datation haute rencontra le scepticisme de R. Demangel, exprimé dans le fascicule suivant des Fouilles de Delphes, consacré à la topographie du sanctuaire de Marmaria, qui parut un an plus tard, en 1926; cet auteur se fondait sur les techniques constructives (scellements en crampons) et sur divers détails architectoniques (hauteur des gouttes du larmier) pour proposer une datation au premier tiers du IV $v^{\mathrm{e}}$. qu'il confina cependant à une note de bas de page ${ }^{27}$.

Tel était l'état de la recherche lorsqu'en 1936, à la suite d'un enchaînement de circonstances qui n'éclaire pas notre propos, l'École française d'Athènes obtint de J. Zay, ministre de l'Instruction Publique, des crédits exceptionnels dont une partie fut consacrée à l'anastylose - c'est-à-dire à la restauration et au remontage - de trois des colonnes extérieures de la tholos. Deux jeunes membres en poste à Athènes, P. Amandry et J. Bousquet, furent chargés de l'ingrate tâche de faire, d'après publi-

20 Mesures extrêmes: 1,105 m et 1,129 m; h. moy. autour de 1,115 m.

21 H.R. Pомтоw, " Die grosse Tholos... », p. 184-185.

22 J. Charbonneaux, FD II 4. La Tholos, p. 4.

23 Pour une introduction à ces questions et une bibliographie, cf. par ex. M.-C. Hellmann, L'architecture grecque, I. Les principes de la construction, 2002, p. 136-137.

24 H.R. Pomtow, "Die grosse Tholos...", p. 185 n. 4.

25 Cf. par exemple J. Charbonneaux, FD II 4. La Tholos, pl. XXVIII et XXX. Nous n'abordons pas ici le problème de la restitution de la toiture qui justifierait à lui seul une étude séparée.

26 J. Charbonneaux, FD II 4. La Tholos, p. 32.

27 R. Demangel, FD II. Le sanctuaire d'Athéna Pronaia (Marmaria). Topographie du sanctuaire, 1926, p. 190 n. 3. 
cation, l'inventaire des tambours disponibles afin de voir ceux dont l'état permettait l'utilisation dans l'anastylose. Grâce notamment à une technique de mesure minutieuse $\mathrm{du}$ fond des cannelures là où $\mathrm{J}$. Charbonneux et $\mathrm{K}$. Gottlob n'avaient mesuré que la hauteur des tambours (il est en effet très difficile de prendre le diamètre des colonnes, car le pentélique se délite verticalement), ils se rendirent compte que les tambours ne se répartissaient pas en quatre groupes, mais en cinq, d'après le diamètre régulièrement décroissant ${ }^{28}$. C'est donc une colonne à cinq tambours, telle qu'on peut encore la voir sur le site, qui fut remontée. La nécessité de préparer la reconstruction par une nouvelle étude avait eu vertu d'archéologie expérimentale et avait conduit à remettre en cause ce qui ne l'avait jamais été auparavant, transformant du même coup un bâtiment qui semblait occuper une place bien définie dans une série en un unicum $^{29}$. La colonne à cinq tambours a une hauteur de 5,928 $\mathrm{m}$, soit un rapport de 6,82 (h/diam. max.).

La manière dont ce changement dans la restitution fut reçu par la communauté scientifique ne manque pas d'intérêt, notamment parce qu'il créait un hiatus entre la datation du monument et son insertion dans les typo-chronologies architecturales. R. Demangel y vit la validation de sa datation vers 370-360: la tholos avait désormais une apparence rien moins que post-parthénonienne ${ }^{30}$. Le caractère plus récent des colonnes justifiait d'adopter une chronologie basse dans la fourchette précédemment définie. Avec une grande prudence, F. Robert se fit l'écho d'une tentation de remettre en cause l'ensemble des datations habituelles ${ }^{31}$. Il s'agissait à nouveau de privilégier, comme Charbonneaux et Gottlob, une datation d'après l'insertion dans les séries chronologiques du rapport hauteur/diamètre inférieur. Plus élancée qu’à Némée, la colonne de Marmaria ne pouvait être comparée qu’à des monuments de la fin du IV et du début du III ${ }^{\mathrm{e}} \mathrm{s}$., " rajeunissant " ainsi d'une centaine d'années! Une autre manière, très minoritaire, de «sauver » la typologie fut de refuser purement et simplement l'adjonction d'un tambour. Si l'idée circule parfois, seul W.B. Dinsmoor l'a publiée ${ }^{32}$ et J.-F. Bommelaer affirmait encore récemment à quel point elle lui semblait impossible ${ }^{33}$. Enfin, dernière manière,

28 P. Amandry, J. Bousquet, "La colonne dorique de la Tholos de Marmaria", Bulletin de correspondance hellénique 64-65 (1940/1), p. 121-124.

29 La légende qui attribue aux marbriers chargés de l'anastylose la découverte de la nécessité d'un tambour supplémentaire, J.-F. Bommelaer (éd.), Marmaria..., p. 59, a été démentie par P. Amandry. Elle n'est pas sans intérêt pour l'histoire des représentations en ce qu'elle opposait savoir abstrait des savants et savoir-faire technique des gens de métier, le second l'emportant sur le premier.

30 R. Demangel, H. Ducoux, "L'anastylose de la Tholos de Marmaria", Bulletin de correspondance hellénique 62 (1938), p. 377.

31 Le ton semble indiquer qu'il se fait plus l'écho de discussions qu'il ne s'engage lui-même dans le débat; F. RoBerT, Thymélè. Recherches sur la signification et la destination des Monuments circulaires dans l'architecture religieuse de la Grèce, 1939, p. 413.

32 W. B. Dinsmoor, The Architecture of Ancient Greece. An Account of its Historic Development, 1950, p. 234 n. 3.

33 J.-F. Bommelaer (éd.), Marmaria..., p. 59-60. 
qui ne manque pas de sel puisqu'elle est celle des inventeurs de la nouvelle restitution, P. Amandry et J. Bousquet, c'est de considérer que la modénature, le décor sculpté et le style des colonnes corinthiennes devaient l'emporter dans la datation du monument: ils proposaient de ne rien changer à la date alors communément admise, " aux alentours de $400^{34}$ ", faisant ainsi de la tholos un bâtiment atypique pour son époque.

Ainsi, il n'est pas toujours aisé de déterminer si c'est une restitution qui permet une datation ou une datation qui entraîne une restitution; l'un et l'autre vont souvent de pair, mais grand est alors le risque de se laisser enfermer dans un raisonnement circulaire.

Néanmoins, à force d'erreurs, de tâtonnements et de corrections, même si des incertitudes demeurent, la restitution du bâtiment dans ses grandes lignes peut être tenue pour acquise. Cependant, la question de la fonction du bâtiment et de son identification demeure ouverte à ce jour. Malgré cela, la communauté scientifique s'accorde aujourd'hui, parmi toutes les propositions avancées, et elles sont nombreuses, à en reconnaître un certain nombre comme erronées ou impossibles. C'est celles-là qui vont nous occuper. Elles ressortissent à deux types de démarches. La première est celle qui entend découvrir la fonction d'un bâtiment à travers son aménagement intérieur. La seconde entend dévoiler un principe de conception qui serait révélateur de la fonction. Souvent, ces démarches s'accompagnent d'interprétations textuelles erronées.

\section{Restitution de l'aménagement intérieur et fonction}

Lors de la redécouverte de la tholos, les archéologues furent frappés par ce qui semblait être une fosse appareillée au centre du monument ${ }^{35}$. Grande fut la tentation d'interpréter ce dispositif comme un bothros, un puits sacrificiel, bien connu par les sources littéraires et déjà attesté dans la " consultation des morts " de l'Odyssée d'Homère qui faisait partie de la culture classique enseignée au lycée. Le bothros, qui plonge dans les entrailles de la terre, est un moyen d'entrer en communication, par le sacrifice, avec les divinités infernales, spécifiquement avec les héros, ces morts à qui l'on rend des honneurs divins. Cette tentation, R. Demangel et F. Poulsen y succombèrent ${ }^{36}$, et ils établirent immédiatement le rapport avec le sanctuaire du héros Phylacos, connu pour être situé dans les environs du sanctuaire d'Athéna grâce à Hérodote et Pausanias ${ }^{37}$. Phylacos, qui avait contribué à sauver Delphes des Perses en 480, méritait bien de recevoir un culte dans un bâtiment aussi prestigieux. La tholos aurait été une châsse destinée

34 P. Amandry, J. Bousquet, "La colonne dorique de la Tholos de Marmaria”, p. 125.

35 J. Charbonneaux, FD II 4. La Tholos, pl. II.

36 F. POUlSEN, "Recherches sur quelques questions relatives à la topographie de Delphes", Bulletin de l'Académie du Danemark (1908) [non vidi], cité par Karo, cf. n. 38.

37 Hérodote, VIII, 36-39; Pausanias, X, 8, 7-8. 
à protéger autant qu’à magnifier un vénérable lieu de culte. G. Karo ${ }^{38}$ démontra, suivi notamment par J. Charbonneaux et K. Gottlob ${ }^{39}$, que le trou au centre du bâtiment était le fruit d'une fouille sauvage à époque tardive. Les parois des dalles contiguës présentent en effet un cadre d'anathyrose qui montre qu'il était recouvert par une dalle. Au demeurant, cette dalle manquante a été retrouvée, elle avait été brisée à la masse, et tant ses dimensions que le matériau dont elle est faite rendent sa restitution indiscutable ${ }^{40}$. De plus, Hérodote place le sanctuaire de ce héros au-dessus de celui d'Athéna, définition à laquelle l'emplacement de la tholos ne semble pas pouvoir correspondre ${ }^{41}$. C'est au nom d'une mauvaise compréhension des vestiges, née de la recherche d'une réalité décrite dans les textes, qu'en retour l'on faisait violence au texte.

Une autre hypothèse d'aménagement de l'espace intérieur de la tholos avait été proposée dès 1904 par Th. Homolle. Il s'agissait de placer au centre du bâtiment un autel rond trouvé dans le voisinage ${ }^{42}$. Il vaut la peine de citer les raisons de la restitution: «L'autel de marbre [...] semble avoir été placé au centre de la tholos. Il est rond comme ce monument, il fut sculpté vers le temps où on le bâtit, et la sculpture est digne des morceaux charmants dont ce temple était décoré ${ }^{43}$. " On voit que c'est sur une vague analogie de style et de forme que l'autel est placé dans la tholos. Le fait qu'il soit également en marbre pentélique a pu influencer Th. Homolle, bien que l'argument ne soit pas invoqué. $\mathrm{H}$. Pomtow accepta cette restitution pour laquelle il fournit une illustration ${ }^{44}$. À cette occasion, il proposa sa propre interprétation du bâtiment ${ }^{45}$, qui fut reprise en 1914 par É. Bourguet dans la première grande synthèse consacrée à Delphes après la Grande fouille ${ }^{46}$. La présence d'un autel circulaire - désormais daté $\mathrm{du} \mathrm{III}^{\mathrm{e}}$ s. et non plus contemporain du bâtiment - dans un bâtiment rond ainsi que d'une banquette courant le long du mur incitait à identifier la tholos avec le prytanée de Delphes et son hestia koinè, son foyer commun connu par les inscriptions. En effet, la double fonction d'un prytanée est d'abriter le foyer de la cité et de servir de lieu de réunion aux magistrats que sont les prytanes. Un bâtiment rond bien connu également appelé tholos, celui de l'Agora d'Athènes, pouvait servir de parallèle et d'antécédent avec d'autant plus de facilité qu'on le savait associé au prytanée. Quant au plan circulaire

38 G. KARO, "En marge de quelques textes delphiques. Suite (1)", Bulletin de correspondance hellénique 34 (1910), p. 218-220.

39 J. Charbonneaux, FD II 4. La Tholos, p. 18.

40 J. Charbonneaux, FD II 4. La Tholos, pl. XXI.

41 S. Settis, "Un'enigma delfico, Pausania, la tholos e il Phyalakeion”, p. 355-377, a repris à son compte cette ancienne proposition d'identification, sans emporter la conviction, cf. L. Lerat, "Les énigmes de Marmaria”, p. 257.

42 Cf. l'étude, très fouillée, de M.-A. ZaGDoun, FD IV, 6 Les reliefs, 1977, p. 79-99 (photographie p. 81).

43 Th. Homolle, "Fouilles de Delphes. Les découvertes de Marmaria", p. 18.

44 H.R. Ромтоw, "Die grosse Tholos...", p. 205-207, pl. II et IV.

45 H.R. Ромтоw, "Die grosse Tholos...”, p. 289 sqq.

46 É. Bourguet, Les ruines de Delphes, p. 324-326. 
du bâtiment, on le croyait, depuis J.G. Frazer ${ }^{47}$, particulièrement lié dans le monde gréco-romain au culte d'Hestia/Vesta dont les travaux de Fustel de Coulanges avaient rappelé l'importance dans la cité grecque. Cette affectation permettait en outre d'expliquer l'irritante énigme du silence de Pausanias au sujet de la tholos: si le Périégète ne la mentionnait pas, c'est qu'il s'agissait d'un bâtiment civil alors qu'il ne s'intéressait qu'aux édifices religieux.

On doit à J. Charbonneaux la réfutation de cette théorie ${ }^{48}$. Tout d'abord, aucune trace sur le dallage ne laisse supposer la présence d'un autel. Au contraire, les dalles centrales sont lisses, de sorte qu'il est probable que rien ne les dérobait à la vue. Ce qui passait pour le banc des prytanes semblait trop haut pour cette fonction, comparé aux autres dispositifs connus, mais se laissait plutôt rapprocher d'une banquette destinée à supporter des statues. Ensuite, les dimensions de la plinthe de l'autel restitué - qui était sans doute carré - rendaient peu probable son insertion dans la tholos. Le parallèle athénien invoqué était trompeur : la bâtiment rond était la salle à manger des prytanes et non le prytanée. En outre, les parallèles de prytanées connus ou supputés à l'époque (à Délos, à Latô, à Olympie) étaient tous de plan carré et comportaient plusieurs pièces. Enfin, Pausanias parle parfois des prytanées. De la théorie tirée par H. Pomtow et É. Bourguet des travaux de Th. Homolle, il ne restait rien ${ }^{49}$. On voit cependant comment, à partir d'une restitution d'aménagement intérieur née d'une observation superficielle et présentée comme un fait matériel peut se construire, par accumulation de parallèles, approximatifs mais illustres, et de généralisations tirées de textes ou de monuments postérieurs, une analyse - très cohérente - qui possède tous les attributs de la démonstration.

\section{Conception et signification}

Pour finir, nous aborderons les interprétations qui, à partir d'une théorie prétendant expliquer la conception générale d'un bâtiment, veulent déduire une fonction et, plus encore, une signification.

H. Thiersch ${ }^{50}$, à partir d'un texte de Plutarque mal interprété, conclut que l'odéon de Périclès à Athènes était de forme circulaire, conclusion qu'il tenta d'élargir à plusieurs rotondes du monde grec, notamment aux tholos de Delphes et d'Épidaure,

47 J. G. Frazer, "The Prytaneum, the Temple of Vesta, the Vestals, Perpetual Fires”, Journal of Philology 14 (1885) p. 145-172.

48 J. Charbonneaux, FD II 4. La Tholos, p. 18-19; J. Charbonneaux, “Tholos et prytanée”, Bulletin de correspondance hellénique 49 (1925), p. 158-178.

49 Seul G. Roux a repris l'idée, sans convaincre, de placer l'autel au centre de la tholos. Néanmoins, cet aménagement aurait été fait très tardivement selon lui, à l'époque où le bâtiment aurait été converti en édifice du culte impérial, G. Roux, Delphes..., p. 206.

50 H. Thiersch, "Antike Bauten für Musik I et II", Zeitschrift für Geschichte der Architektur 2 (1908-1909), p. 71-72. 
situées dans des sanctuaires où se déroulaient des concours musicaux. Un essai de comparatisme l'amena à penser que cette forme était particulièrement adaptée aux édifices de spectacle (il invoque pour son propos, photographies à l'appui, une piste de danse contemporaine et un kiosque à musique ${ }^{51}$ ). Rien n'indique une telle destination pour la tholos. De plus, le bâtiment semble trop luxueux pour être un édifice de spectacle et il fut facile d'ironiser sur la musique de chambre que l'on y jouait, vu la faiblesse du public qui aurait pu assister aux représentations. Cette théorie, "ingénieuse sinon vraisemblable " pour reprendre le mot de J. Charbonneaux ${ }^{52}$, fut ruinée par la fouille de l'odéon de Périclès qui montra que le monument sur lequel on prétendait la fonder était en fait quadrangulaire et elle fut abandonnée par son auteur même.

Plus récente et mieux étayée en apparence, la théorie de F. Robert visait à voir dans les édifices circulaires ou absidaux du monde grec des monuments de type chtonien: "Que la rotonde de Marmaria, la rotonde d'Épidaure, et l'Arsinoéion, soient tout ensemble exceptions, merveilles, et énigmes, n'est-ce point un groupement d'indices cohérents et suggestifs? Quelque pensée religieuse, unique et importante, n'a-t-elle point dicté cette permanente association de somptuosité et de mystère? [nous soulignons] ${ }^{53}$." Remarquons comment, à partir d'un double postulat: "Le circulaire est chtonien " et "tous les monuments circulaires ne peuvent avoir qu'une signification commune ", on peut parvenir à trouver ce que l'on cherche. F. Robert part de l'autel rond que nous avons vu précédemment. Il est creux, donc en contact avec la terre, donc chtonien. Il considère alors comme démontré qu'il y avait une dimension chtonienne dans le culte d'Athéna, alors même que d'autres cultes que celui de la déesse sont présents sur la terrasse. Pour lui, " on doit nécessairement conclure de là que la tholos, dont nous ignorons l'usage, a reçu sa forme circulaire par allusion directe à des rites chtoniens qui se célébraient encore ou qui avaient été célébrés jadis dans le sanctuaire d'Athéna [nous soulignons] »Et après avoir rappelé que la tholos n'est pas un hérôon, «il ne reste plus qu’une hypothèse possible, et c'est celle de MM. Ch. Picard et P. de la Coste-Messelière: un culte chtonien féminin aurait précédé celui de l'Athéna olympienne, et c'est de ce culte que la Tholos porterait témoignage [nous soulignons] ». La découverte de statuettes féminines mycéniennes dans les fouilles du sanctuaire venait encore étayer cette théorie ${ }^{54}$.

La dimension chtonienne de la tholos n'est plus soutenue par personne depuis une cinquantaine d'années; elle est difficile à réfuter, car elle est également impossible à démontrer. Les arguments sur lesquels elle repose sont des plus faibles: les statuettes en question sont aujourd'hui interprétées comme des dépôts d'offrandes funéraires perturbés par les travaux de construction antiques. Quant à l'autel, il est plus récent de deux siècles au moins que la tholos et n'a rien de spécifiquement chtonien. Il est intéressant

51 H. Thiersch, “Antike Bauten für Musik I et II", ill. 17 et 18.

52 J. Charbonneaux, FD II 4. La Tholos, p. 28.

53 F. Robert, Thymélè..., p. 13.

54 F. Robert, Thymélè..., p. 414-420. 
d'observer la démarche de l'auteur: présence des deux postulats mentionnés plus haut, "sur-sollicitation " d'indices disparates éloignés chronologiquement les uns des autres et dont le contexte archéologique est problématique, les statuettes et un autel. Ces indices sont mis au service de sa croyance en des modèles d'explication évolutionnistes généraux venus de l'histoire des religions, tel que la succession de divinités chtoniennes et ouraniennes. Enfin, il utilise une rhétorique qui relève plus de la conviction que de la démonstration: "nécessairement ", "plus qu'une hypothèse possible ». Remarquons qu'a pu jouer chez un homme relativement jeune (entré à l'ÉfA en 1932) la volonté de généraliser et de démontrer à tout prix, dans une démarche qui se voulait scientifique et définitive, ce qui avait été chez ses maîtres une intuition. La mention de Ch. Picard, ancien directeur de l'École, et de P. de La Coste Messelière qui exerça une forte influence sur la plupart des Delphiens de cette époque, est significative et leur pensée irrigue tout l'ouvrage.

Le point commun de ces deux méthodes est qu'elles ont procédé par extrapolation, sans tenir aucun compte des particularités de chacun des monuments étudiés, ceci au nom d'une théorie générale fondée sur quelques exemples disparates interprétés souvent de manière abusive et érigés en série. L'idée que tous les bâtiments d'un même plan doivent s'interpréter de la même manière et que la conception d'un monument vaut signification justifie tous les égarements, puisque tout silence semble confirmer la théorie et qu'on ne s'embarrasse guère de chronologie.

À passer en revue ces différentes erreurs sur la tholos, on peut être saisi de trouble en constatant que, dans la plupart des cas, ce n'est pas la démarche mise en œuvre qui est la cause de la faillite du raisonnement scientifique, mais plutôt la mauvaise application, naïve et péremptoire, de pratiques normalement en vigueur pour restituer, dater et identifier les monuments: trouver des parallèles, chercher dans les textes, partir de la forme, partir de l'aménagement intérieur considéré comme un indice de la fonction... Dans le cas de la tholos toutefois, ces démarches sont biaisées parce que nous manque le sens qui permettrait d'interpréter en toute certitude les indices matériels repérés et que, bien souvent, la construction du sens est précisément le postulat de départ que l'on va démontrer: ainsi en est-il par exemple de la prétendue signification chtonienne de la tholos, ou encore de la restitution de la tholos en prytanée... la circularité du raisonnement s'appuie de surcroît sur une observation peu rigoureuse. La figure de H. Pomtow est emblématique de ce cas jusqu'à la caricature: il avait d'abord renoncé à étudier la tholos faute de temps, puisque son architecte Wenzel lui avait affirmé que l'étude nécessitait le travail de deux architectes pendant 4 à 5 jours. Mais, à la suite d'une petite découverte, c'est en deux séances de 40 minutes de travail chacune qu'ils firent le relevé du bâtiment ${ }^{55}$. Il n'y a plus lieu de s'étonner s'ils ne surent pas voir les traces de la présence d'une colonnade intérieure. L'esprit de système qui extrapole les données lorsqu'elles n'existent pas est également cause d'une série d'erreurs; spécifiquement,

55 H.R. Pomtow, "Die grosse Tholos...", p. 182 n. 1. 
l'approche idéaliste de l'histoire des religions et l'approche symboliste de l'architecture se sont révélées être des voies particulièrement aventureuses: à ne pas tenir compte de l'observation, on trouve toujours ce que l'on cherche. Les errances sur la tholos sont ainsi emblématiques des pratiques ordinaires de l'archéologie, mais témoignent aussi des limites de la possibilité d'interprétation.

La hâte ou la recherche du sensationnel s'imposent comme d'autres grandes causes d'erreur. Elles peuvent s'expliquer par l'insertion de l'érudit dans son milieu. Le travail du savant peut être ainsi affecté par des enjeux socioprofessionnels ou politiques. Revenons à H. Pomtow: professeur de lycée, il se vit confier par l'académie de Prusse la charge de réaliser le corpus des inscription de Phocide et milita pour que l'Empire allemand entreprenne des fouilles sur le site du sanctuaire d'Apollon Pythien ${ }^{56}$. Il se rêva sans doute comme l'homme de Delphes. Son rêve s'écroula lorsque la France se vit accorder le monopole de l'exploration archéologique et de la publication sur le site pendant dix ans. À l'expiration de ce délai et avec l'accord du service archéologique grec, il revint à Delphes et entreprit de publier le résultat des fouilles françaises qui n'étaient plus protégées par un monopole. Cette course pour être le premier à publier est particulièrement sensible pour la tholos et le poussa à travailler vite et mal ${ }^{57}$. Certaines de ces remarques peuvent s'appliquer également au cas de F. Robert évoqué plus haut.

Ainsi, l'étude de l'erreur, en plus d'une vertu prophylactique relative, certes, mais réelle, permet d'esquisser une histoire de la pensée et de la pratique d'un milieu social, celui des chercheurs, des savants, des universitaires, pour laquelle elle offre une entrée. Elle peut également apporter une contribution à l'histoire culturelle: à travers les erreurs, c'est l'histoire des passions d'une époque qui se révèle à nous.

\begin{tabular}{l} 
Nicolas KYRIAKIDIS \\
\hline Université de Strasbourg - École française d'Athènes \\
EfA \\
Didotou 6 \\
10680 Athènes (Grèce) \\
nicolas.kyriakidis@efa.gr
\end{tabular}

56 Pour un premier aperçu sur le sujet, sans fond, des querelles entre Pomtow et les archéologues français, cf. Collectif (éd.), La redécouverte de Delphes, p. 198-199.

57 Voir par exemple le ton polémique de H.R. Pomtow, "Die grosse Tholos...", p. $180-182$. 


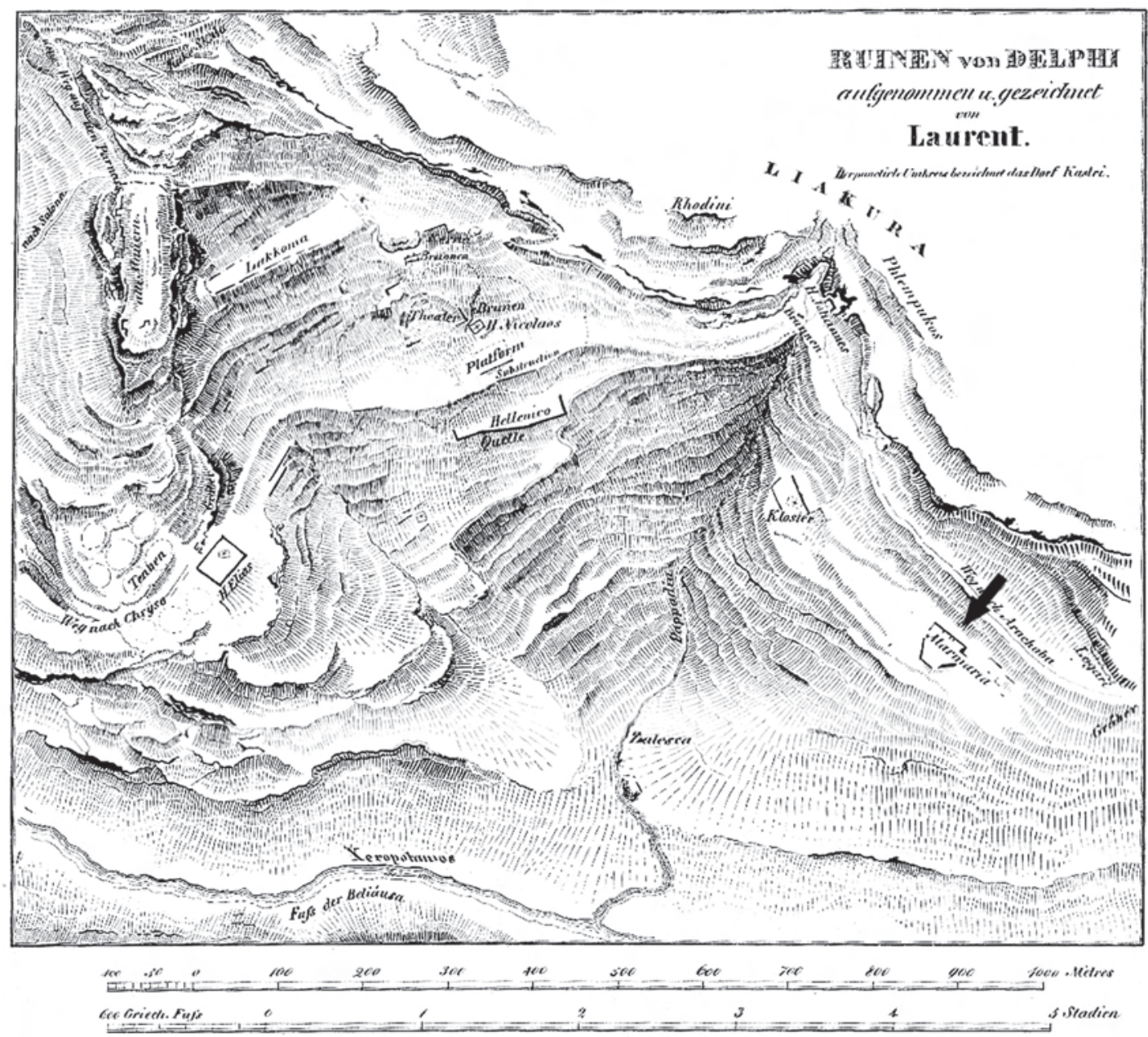

Fig. 1 : site de Delphes, plan des vestiges antiques relevés par Laurent (1838) in H. N. UlRICHS, Reisen und Forschungen, vol. I, deuxième planche hors texte. 


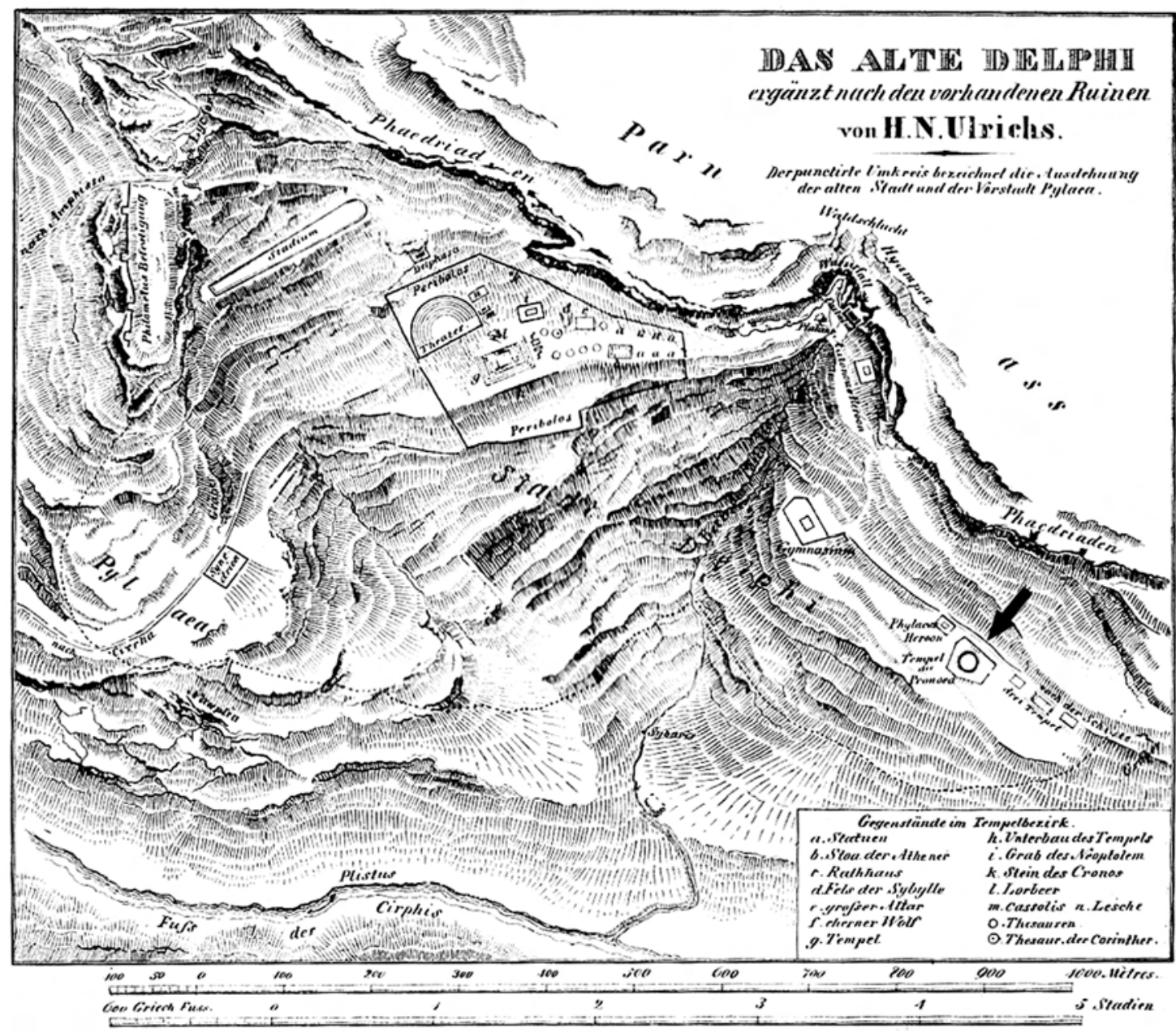

Fig. 2: site de Delphes, plan restitué par Ulrichs (1840) in H.N. UlRICHS, Reisen und Forschungen, vol. I, première planche hors texte. 